\title{
Can Simple Preoperative Hemoglobin Testing Screen Symptomatic Anemia in Patients Undergoing Ambulatory Surgeries in Third World Countries?
}

\author{
Sobia Khan ${ }^{1}$, Mueen Ullah Khan ${ }^{2}$, Khalid Samad ${ }^{1}$ \\ ${ }^{1}$ Department of Anesthesia, Aga Khan University Hospital, Karachi, Pakistan; ${ }^{2}$ Department of Anesthesia, King Khalid University \\ Hospital, Riyadh, KSA. \\ Email: sobia.khan@aku.edu, khalid.samad@aku.edu
}

Received June $19^{\text {th }}, 2012$; revised July 21 ${ }^{\text {st }}, 2012$; accepted August $10^{\text {th }}, 2012$

\begin{abstract}
Background: Patients coming for ambulatory surgeries are mostly healthy adults and asymptomatic anemia in these patients is rare. According to international standards, perioperative period is not an appropriate setting to screen and investigate the cause of asymptomatic anemia, but in third world countries where iron deficiency is rampant, it is generally required as per local hospitals policy to test hemoglobin levels prior to any surgery in order to prevent morbidity. The purpose of our study is to look at the prevalence of anemia in patient undergoing minor elective ambulatory surgeries. Method: This was a cross sectional observational study conducted at tertiary care unit, Karachi, Pakistan. A total of 385 ASA-I (American Society of Anesthesiologist) and II patient's age ranged 18 - 60 years, scheduled for day care surgical procedures were enrolled in the study. Results: Anemia was detected in 74 (19.2\%) patients and its prevalence was found to be higher in females and in patients above 50 years of age. However, the presence of anemia did not have any influence on the perioperative outcomes or management. Conclusion: The routine preoperative hemoglobin testing does not have any effect on the perioperative outcomes in asymptomatic patients who are planned for elective day care surgeries.
\end{abstract}

Keywords: Hemoglobin; Anemia; Day Care Surgery

\section{Introduction}

The pre-anesthetic evaluation is defined as the process of clinical assessment that precedes the delivery of anesthesia care for surgery and for non-surgical procedures. The ultimate goal of preoperative medical assessment is to reduce the morbidity associated with surgery and anesthesia and to increase the quality and decrease the cost of perioperative care. It also serves the purpose of optimizing patients for elective surgery and if necessary, implements measures to prepare higher risk patients for surgery $[1,2]$.

The pre-operative anesthesia evaluation comprises of comprehensive history, physical examination and laboratory tests [1]. The history should include a complete review of systems, medication history, surgical and anesthetic history, history of allergies and functional status. The physical examination and laboratory tests should be focused on information gathered from the history [1-6].

Recent literature suggests that preoperative laboratory testing should be based on clinical examination rather than routine protocols [1-6]. Preoperative hemoglobin test or screening for anemia is included in most of the hospitals preoperative testing protocols in third world countries despite the fact that routine laboratory tests for all patients are not good screening device for diseases [3].

It is important to note that the Normal test values are arbitrarily defined as those occurring within two standard deviations from mean thereby insuring that five percent of healthy individuals who have a single screening test will have an abnormal result, as more tests are ordered, the likelihood of false positive tests increases. These unexpected abnormal results have very little impact on the anesthetic management if at all [4,6-9].

At our hospital, despite knowing the current international guidelines, we routinely order hemoglobin levels for all patients at the time of pre-anesthesia evaluation regardless of patient's age and kind of surgery because the World Health Organization has identified that iron and folic acid deficiency is a severe problem in Pakistan and it has devastating impacts on health and survival [10].

The objective of our study was to determine the prevalence of anemia and to observe the impact of anemia in terms of case cancellation, case rescheduling, un- 
planned blood transfusion and unplanned hospital admission due to evaluation/correction of anemia or to treat the perioperative complications attributable to anemia in patients fulfilling our inclusion criteria for study.

\section{Methods}

This study was conducted over a period of six months. A total of 385 patients were recruited by a non-probability consecutive sampling technique from preoperative anesthesia clinic at tertiary care hospital of Karachi, Pakistan.

Hospitals ethical review committee exempted the study for need of ethical approval and informed patient consent because it was a cross sectional observational study.

All the patients without any systemic co-morbids were labeled as ASA-I (American Society of Anesthesiologist Grade I) while patients with systemic co morbid or disease which was under control were labeled as ASA-II (American Society of Anesthesiologist Grade II) in accordance with international definitions.

ASA-I and ASA-II patients falling in age group 18 to 60 years who visited preoperative anesthesia clinic to be evaluated before elective day care surgical procedures which included but were not limited to herniorraphy, fistulectomy, septoplasty, mastoidectomy, hemorrhidectomy and cataract surgery were enrolled in the study.

The patients with diseases requiring regular blood transfusions (e.g. patients with thalassemia major, sickle cell anemia), patients who either donated blood or received blood transfusion within 4 months and patients with chronic hepatic, renal or cardiac disease were excluded.

Once enrolled the patients were evaluated and investigated as per our departmental protocol by taking detailed medical history, performing relevant physical examination and doing laboratory tests which included baseline hemoglobin and hematocrit level for ALL patients regardless of their age and planned surgical procedure.

We considered male patients as anemic if their hemoglobin levels were less than $13 \mathrm{gdl}^{-1}$ and females were labeled as anemic if their hemoglobin levels were less than $11 \mathrm{gdl}^{-1}$ in accordance with the our hospitals laboratory normograms [11].

All these patients were followed after the admission in the day care suite; their whole intraoperative and postoperative course was monitored by reviewing their charts on the same day by the primary investigator.

Any untoward incidents like case postponement or case cancellations (by the primary anesthetist who was not the primary investigator) as a result of low hemoglobin, unplanned perioperative blood transfusion or unplanned post-surgery hospital admission (which was related to low hemoglobin) was noted.
The relevant details were documented by primary investigator in a predesigned form.

We considered anticipated frequency of anemia as 10 percent with an absolute precision of three percent and a level of significance of 5 percent. Data was analyzed by using SPSS (Statistical Package for Social sciences) Version 16. Frequency and percentage was computed for qualitative variables like gender, ASA physical status. Mean and standard deviation was computed for quantitative variables like age, weight, and hemoglobin level. Student $t$ test was applied to compare quantitative variables. 95 percent confidence interval for the frequency in percent was computed for anemia. In all cases P-value of less than 0.05 was considered as statistically significant.

\section{Results}

Total of 385 patients were studied during six month period that were anesthetized in surgical day care unit for day case surgeries. 254 (66\%) were males and 131 (34\%) were females (Table 1).

Age range of male patients was $22-50$ years ( $36 \pm 12$ ) and of female patients was $18-46$ years $(32 \pm 11) .230$ (59.7\%) patients were labeled as ASA-I and 155 (40.3\%) were labeled as ASA-II by the primary anesthetist.

Among 230 ASA-I patients 156 (68\%) were males while $74(32 \%)$ were females $\left(\chi^{2}=0.87\right.$; $\mathrm{DF}=1, p$-value $=0.350143)$ (Table 1).

The mean age was $31 \pm 10$ years in ASA-I patients. The frequency of anemia was $29(12.6 \%)$ in ASA-I patients (Graph I). The mean hemoglobin came out to be $13.6 \pm 1.5 \mathrm{gdl}^{-1}$.

Among 155 ASA-II patients 98 (63\%) were males while 57 (37\%) were females. The mean age in ASA-II patients was $39 \pm 13$ years. The frequency of anemia was 45 (29\%). The mean hemoglobin was $12.8 \pm 2 \mathrm{gdl}^{-1}$ in ASA-II patients.

The mean hemoglobin in the study population was found to be $13.9 \mathrm{gdl}^{-1}$. The maximum hemoglobin level was $16 \mathrm{~g} / \mathrm{dl}$ and lowest was $5 \mathrm{~g} / \mathrm{dl}$. The overall mean hemoglobin in males was $14 \mathrm{gdl}^{-1}$ while in females it was $11.9 \mathrm{gdl}^{-1}$.

Table 1. Breakup of number of patients according to ASA level and gender.

\begin{tabular}{ccc}
\hline ASA Level & Number of Patients & Mean \pm SD \\
\hline I & 230 & $31.65 \pm 10.621$ \\
II & 155 & $39.53 \pm 12.917$ \\
Gender & Number of Patients & Mean \pm SD \\
M & 254 & $36.02 \pm 12.375$ \\
F & 131 & $32.50 \pm 11.590$ \\
\hline
\end{tabular}


Overall frequency of anemia was 74 (19.2\%). None of the case was cancelled/rescheduled because of anemia except for one patient with Hemoglobin of $5 \mathrm{~g} / \mathrm{dl}$. None of the patient had unexpected blood loss perioperatively and there were no incidence of perioperative transfusion as well. All the patients were discharged home as per schedule.

\section{Discussion}

Advancement of surgical technology, availability of sophisticated instruments and monitoring tools have made surgery and anesthesia less complicating and threatening to the patients physiology, allowing early and safe recovery. All of this has led to the change and many procedures are now scheduled as day case procedures. Minimally invasive procedures have reduced the importance of routine preoperative investigations in clinically healthy individuals.

Despite this, screening for anemia and determination of hemoglobin percentage prior to any surgery is an integral part of preanesthetic evaluation in many institutes especially of third world countries including ours. The rationale is more of a custom than valid scientific evidence [12-14].

Anesthesiologist is responsible to ensure the adequate supply of oxygen to all the vital organs during surgical procedures and under anesthesia. ASA task force has stressed upon evaluation of vital organs perioperatively for adequate oxygenation and perfusion [14]. The heart rate, blood pressure, oxygen saturation, urine output and electrocardiography are the preoprative conventional tools of monitoring.

The total oxygen availability to the tissues depends on the product of cardiac output and oxygen content. The main determinant of oxygen can be augmented in the preoperative period for enhancing the availability of oxygen [14]. This has been the basis for preoperative hemoglobin determination. General anesthesia places patients at risk of hypoxemia secondary to decreased functional residual capacity in the lungs. Therefore some margin of safety is warranted during general anesthesia.

In South Asian underdeveloped countries where anesthetists ask for preoperative hemoglobin in all patients because most of the patients never had any laboratory investigation previously. They think this may be the best chance for doing some basic baseline investigations in all patients coming for surgery.

Not only short surgical procedures involve minimal risk but there has also been a change in transfusion thresholds. According to the ASA consensus the transfusion is not necessarily indicated in a patient with hemoglobin as low as $7 \mathrm{gdl}^{-1}$ if that patient is preoperatively asymptomatic, normovolemic, and no further intraoperative blood loss is anticipated [14].

As most of literature for individual preoperative investigation is from developed countries therefore in this study we aimed to find out the prevalence of anemia in patients coming for day case procedures at our setup and to assess if routine hemoglobin tests are warranted for those asymptomatic individuals. At our tertiary care hospital, patients from different ethnicity and parts of country are catered. The prevalence of anemia in underdeveloped countries like ours is high due to nutritional deficiencies. In our study of 385 patients $19.2 \%$ were found to be anemic but none of them had any untoward incident intraoperatively, none had surgery cancellation, rescheduling, or unexpected blood transfusion.

Pal K. M. et al. [15] retrospectively reviewed 320 patient's case notes who attended the general surgical department for common surgical procedures. They found that the prevalence of anemia in $19.4 \%$ patients, and anemia was more common in females as compare to males. These results are consistent with the studies performed at different time period and with our results.

Our study has shown that doing preoperative hemoglobin in all patients is not justified as it does not compromise the safety of the individuals coming for minor surgical procedures under anesthesia.

The strength of our study is that the sample size is good enough to enhance the power of study. Moreover the frequency of anemia in our results is consistent with another retrospective study done few years ago at our tertiary care hospital [15]. The results of our study are consistent with the conclusion of other international studies that preoperative hemoglobin testing in clinically healthy individuals is unnecessary provided the proposed surgery is a minor day care procedure. However we did recognize few limitations of our study as well. Although all the enrolled patients were scheduled for the day care procedures there was a variation in type of surgical procedures which may have affected the outcome. Secondly the patients were assigned ASA status by the primary anesthetist instead of the primary investigator; this could have contributed to a bias. Lastly pediatric population was not enrolled in the study, although this is the group where anemia is known to occur more frequently. As a result the actual frequency of anemia in all the patients coming for day care surgical procedures may be higher.

After results compilation and interpretation we forwarded our suggestions to our departmental Committee for revising our departments existing guidelines/policies in accordance with locally available data and internationally available literature. The suggestions will also be carried forward to the national forum of Pakistan Society of Anesthesiologist to benefit the general Pakistani popula- 
tion.

We conclude that the routine preoperative hemoglobin estimation for elective day care surgical procedures in asymptomatic healthy adult patients is unnecessary as has also been shown by international literature $[16,17]$. The anemia although frequent in our population can be judged by a good history which must include the questions about socioeconomic status apart from the other relevant details and thorough clinical examination. ASA-I and ASA-II cases can be waived off the routine preoperative hemoglobin testing. Only individuals in whom the clinical history indicates or physical examination reveals anemia should have the preoperative blood test to document the level of hemoglobin. Moreover anemia even if present neither affect the safety nor it changes the periopertive management in case of day care surgical procedures under anesthesia provided strict international standards of monitoring are maintained throughout both intra and postoperative course.

\section{Acknowledgements}

We would like to express our gratitude to Mr. Iqbal Azam Assistant Professor, Community Health Sciences and to the nursing staff, day care suite, Aga Khan University Hospital for providing guidance and assistance in the completion of this work.

\section{REFERENCES}

[1] F. A. Michota and S. D. Frost, "The Preoperative Evaluation: Use the History and Physical Rather than Routine Testing," Cleveland Clinic Journal of Medicine, Vol. 71, No. 1, 2004, pp. 63-70. doi:10.3949/ccjm.71.1.63

[2] F. Y. Ajimura, A. S. Maia, A. Hachiya, A. S. Watanabe, P. N. Mdo, A. M. Mde and F. S. Machado, "Preoperative Laboratory Evaluation of Patients Aged over 40 Years Undergoing Elective Non-Cardiac Surgery," Sao Paulo Medical Journal, Vol. 123, No. 2, 2005, pp. 50-53. doi:10.1590/S1516-31802005000200003

[3] American Society of Anesthesiologists Task Force on Preanesthesia Evaluation, "Practice Advisory for Preanesthesia Evaluation: A Report by the American Society of Anesthesiologists Task Force on Preanesthesia Evaluation,” Anesthesiology, Vol. 96, No. 2, 2002, pp. 485-496. doi:10.1097/00000542-200202000-00037

[4] M. S. King, "Preoperative Evaluation,” American Family Physician, Vol. 62, 2000, pp. 387-396.

[5] G. H. Tabas and M. S. Vanek, "Is 'Routine’ Laboratory Testing a Thing of the Past? Current Recommendations
Regarding Screening,” Postgraduate Medicine, Vol. 105, No. 3, 1999, pp. 213-220. doi:10.3810/pgm.1999.03.639

[6] R. K. Johnson and A. J. Mortimer, "Routine Preoperative Blood Testing: Is It Necessary?” Anesthesia, Vol. 57, No. 9, 2002, pp. 914-917. doi:10.1046/j.1365-2044.2002.02750.x

[7] R. H. Haug and R. L. Reifeis, "A Prospective Evaluation of the Value of Preoperative Laboratory Testing for Office Anesthesia and Sedation,” Journal of Oral and Maxillofacial Surgery, Vol. 57, No. 1, 1999, pp. 16-20. doi:10.1016/S0278-2391(99)90623-7

[8] S. Dzankic, D. Pastor, C. Gonzalez and J. M. Leung, “The Prevalence and Predictive Value of Abnormal Preoperative Laboratory Tests in Elderly Surgical Patients,” Anesthesia \& Analgesia, Vol. 93, No. 2, 2001, pp. 301-308.

[9] M. S. Mallick, "Is Routine Preoperative Blood Testing in Children Necessary?” Saudi Medical Journal, Vol. 27, No. 12, 2006, pp. 1831-1834.

[10] “ADB to Pilot Project to Combat Nutritional Problems," 2006. http://www.adb.org/Media/Articles/2006/9538

[11] I. Bates and S. M. Lewis, "Practical Hematology," Churchill Livingstone, Philadelphia, 2001.

[12] S. Mantha, M. F. Roizen, J. Madduri, Y. Rajendar, S. K. Naidu and K. Gayatri, "Usefulness of Routine Preoperative Testing," Journal of Clinical Anesthesia, Vol. 17, No. 1, 2005, pp. 51-57. doi:10.1016/j.jclinane.2004.04.002

[13] J. Carlislie, J. Langham, G. Thomas and I. Editorial, "Guidelines for Routine Preoperative Testing," British Journal of Anaesthesia, Vol. 93, No. 4, 2004, pp. 495-497. doi:10.1093/bja/aeh230

[14] American Society of Anesthesiologists Task Force on Perioperative Blood Transfusion and Adjuvant Therapies, "Practice Guidelines for Perioperative Blood Transfusion and Adjuvant Therapies: An Updated Report by American Society of Anesthesiologist Task Force on Perioperative Blood Transfusion and Adjuvant Therapies," Anesthesiology, Vol. 105, 2006, pp. 198-208.

[15] K. M. Pal, I. A. Khan and B. Safdar, "Preoperative Work up: Are the Requirements Different in a Developing Country?” Journal of Pakistan Medical Association, Vol. 48, No. 11, 1998, pp. 339-341.

[16] B. J. Narr, M. E. Warner, D. R. Schroeder and M. A. Warner, "Outcomes of Patients with No Laboratory Assessment before Anesthesia and a Surgical Procedure," Mayo Clinic Proceedings, Vol. 72, No. 6, 1997, pp. 505509. doi:10.4065/72.6.505

[17] F. Chung, H. Yuan, L. Yin, S. Vairavanathan and D. T. Wong, "Elimination of Preoperative Testing in Ambulatory Surgery,” Anesthesia \& Analgesia, Vol. 108, No. 2, 2009, pp. 467-475. doi:10.1213/ane.0b013e318176bc19 